\title{
The Blessing and the Curse of the Multiplicative Updates
}

\author{
Manfred K. Warmuth ${ }^{\star}$ \\ Computer Science Department \\ University of California, Santa Cruz \\ CA 95064, U.S.A. \\ manfred@cse.ucsc.edu
}

\begin{abstract}
Multiplicative updates multiply the parameters by nonnegative factors. These updates are motivated by a Maximum Entropy Principle and they are prevalent in evolutionary processes where the parameters are for example concentrations of species and the factors are survival rates. The simplest such update is Bayes rule and we give an in vitro selection algorithm for RNA strands that implements this rule in the test tube where each RNA strand represents a different model. In one liter of the RNA "soup" there are approximately $10^{20}$ different strands and therefore this is a rather high-dimensional implementation of Bayes rule.

We investigate multiplicative updates for the purpose of learning online while processing a stream of examples. The "blessing" of these updates is that they learn very fast because the good parameters grow exponentially. However their "curse" is that they learn too fast and wipe out parameters too quickly. We describe a number of methods developed in the realm of online learning that ameliorate the curse of these updates. The methods make the algorithm robust against data that changes over time and prevent the currently good parameters from taking over. We also discuss how the curse is circumvented by nature. Some of nature's methods parallel the ones developed in Machine Learning, but nature also has some additional tricks.
\end{abstract}

^ Supported by NSF grant IIS-0917397. 